\title{
Análise da rede social de um Centro de Atenção Psicossocial Álcool e Drogas*
}

Analysis of the social network of Center for Psychosocial Attention for alcohol and other drugs

Análisis de la red social de un Centro de Atención Psicosocial para el alcohol y las drogas

\section{Débora Schlotefeldt Siniak ${ }^{\mathrm{I}}$, Leandro Barbosa de Pinho ${ }^{\mathrm{II}}$, João Nunes Maidana Júnior ${ }^{\mathrm{III}}$, Marciele Barcelos Ávila ${ }^{\mathrm{IV}}$, Vanessa Alvez Mora da Silvav}

\begin{abstract}
Resumo: Objetivo: investigar a rede social de um Centro de Atenção Psicossocial para álcool e outras drogas (CAPS AD) a partir dos encaminhamentos e recebimentos de casos. Método: quantitativo, descritivo, realizado em um município do Estado do Rio Grande do Sul, Brasil. A coleta de dados ocorreu de agosto a dezembro de 2017, por meio de roda de conversa, aplicação de questionário em formato de Escala Likert e construção de sociogramas, analisadas pela Análise de Redes Sociais; participaram do estudo 13 profissionais. Resultados: havia 37 atores envolvidos de forma direta ou indireta no cuidado às pessoas que usam drogas. O CAPS AD e a Estratégia Saúde da Família são alguns dos principais serviços que encaminham e recebem demandas dessa população. Conclusão: são necessárias a descentralização da rede e conectividade entre os atores, e pensar em formas de atuação conjunta que propiciem a construção de um sistema integrado e cooperativo.

Descritores: Saúde Mental; Serviços de Saúde Mental; Assistência à Saúde; Rede Social; Transtornos Relacionados ao Uso de Substâncias
\end{abstract}

Abstract: Objective: to investigate the social networking of a Center for Psychosocial Attention for alcohol and other drugs (CAPS AD) from referrals and case receipts. Method: quantitative, descriptive, developed in a municipality of the State of Rio Grande do Sul, Brazil. Data was collected from August to December 2017, by means of a yarning circle, application of a questionnaire in a Likert Scale format, and construction of sociograms, reviewed by Social Networking Analysis; 13 professionals participated in the research. Results: there were 37 actors directly or indirectly involved in caring for people who use drugs. CAPS AD and the Family Health Strategy are

\footnotetext{
${ }^{\text {I }}$ Enfermeira, Doutora em Enfermagem, Docente do Curso de Enfermagem da Universidade Federal do Pampa, Uruguaiana, RS, Brasil. E-mail: deborasiniak@gmail.com, ORCID: 0000-0002-7689-6953

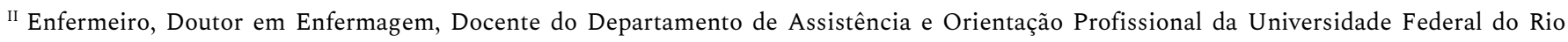
Grande do Sul, Porto Alegre, RS, Brasil. E-mail: lbpinho@ufrgs.br, ORCID: 0000-0003-1434-3058

III Enfermeiro, Mestrando do Programa de Pós-Graduação em Enfermagem da Universidade Federal do Rio Grande do Sul, Porto Alegre, RS, Brasil. E-mail: juniordana@hotmail.com, ORCID: 0000-0001-8411-5566

IV Enfermeira, Mestranda do Programa de Pós-Graduação em Enfermagem da Universidade Federal do Rio Grande do Sul, Porto Alegre, RS, Brasil. E-mail: marcieleba@yahoo.com.br, ORCID: 0000-0003-0119-5471.

$\checkmark$ Enfermeira, Doutoranda do Programa de Pós-Graduação em Enfermagem da Universidade Federal do Rio Grande do Sul, Porto Alegre, RS, Brasil. E-mail: valvez_enfermagem@hotmail.com, ORCID: 0000-0003-0235-7663
}

* Extraído da tese: A organização da rede a partir de um centro de atenção psicossocial para o cuidado ao usuário de drogas. Programa de Pósgraduação em Enfermagem. Universidade Federal do Rio Grande do Sul, 2018. 
some of the main services that refer and receive demands from this population. Conclusion: Decentralization of the network and connectivity among the actors are necessary, and ways of working together to build an integrated and cooperative system must be considered.

Descriptors: Mental Health; Mental Health Services; Delivery of Health Care; Social Networking; Substance-related Disorders

Resumen: Objetivo: Investigar la red social de un Centro de Atención Psicosocial para el alcohol y otras drogas (CAPS AD) a partir de las remisiones y recepciones de casos. Método: cuantitativo, descriptivo, realizado en un municipio del Estado de Rio Grande do Sul, Brasil. La recopilación de datos tuvo lugar de agosto a diciembre de 2017, mediante una ronda de conversación, la aplicación de un cuestionario en formato de escala Likert y la construcción de sociogramas, analizados por el Análisis de Redes Sociales; 13 profesionales participaron en el estudio. Resultados: había 37 actores involucrados directa o indirectamente en el cuidado de las personas que consumen drogas. CAPS AD y la Estrategia de Salud Familiar son algunos de los principales servicios que atienden y reciben las demandas de esta población. Conclusión: es necesario descentralizar la red y la conectividad entre los actores, y pensar en formas de actuar juntos para construir un sistema integrado y cooperativo.

Descriptores: Salud Mental; Servicios de Salud Mental; Prestación de Atención de Salud; Red Social; Trastornos Relacionados con Sustancias

\section{Introdução}

O debate sobre as drogas é multidimensional, exigindo novos modos de operar nos serviços que prestam assistência a estas populações, ampliando a abordagem ao usuário, considerando sua família, suas redes. ${ }^{1-2} \mathrm{O}$ tema também impõe desafios à garantia de que esses serviços, quando implantados, não se cristalizem em pontos isolados de atenção. As transformações do modelo de atenção em saúde mental fomentadas pelo acúmulo teórico das políticas públicas brasileiras convocam a transformar paradigmas, tanto no campo da saúde mental quanto acerca de usos e abusos de drogas. ${ }^{3}$

Apesar dos amplos movimentos de desinstitucionalização no âmbito da saúde mental, os usuários de drogas ainda são atravessados por valores moralistas e estigmatizantes internalizados na cultura brasileira, o que vê nesse usuário é alguém que não pode conviver em sociedade. Em vista disso, busca-se ampliar o trânsito nos espaços da rede e suas potências por meio de arranjos que visem à inserção sociocultural e ao cuidado integral em saúde. ${ }^{4}$ 
Nesse sentido, os serviços de saúde são organizados em redes de complexidade crescente, interligados entre si por meio de processos formais de referência/contrarreferência. Utilizar instrumentos de referência e contrarreferência representa uma importante ferramenta para a melhoria na integração dos serviços de saúde. ${ }^{5}$

Contudo, um legítimo trabalho em rede deve acontecer de forma comprometida, por meio do estabelecimento de trocas de cuidado, informações, flexibilidade e ações conjuntas entre os atores. Para além de simples encaminhamentos, a rede precisa articular ações setoriais e intersetoriais no território, atendendo de forma efetiva às necessidades sociais e de saúde. ${ }^{6} \mathrm{~A}$ articulação em rede trata, portanto, de um desafio aos serviços, de modo a superar esses obstáculos para sua efetivação..$^{7-8}$

Ao encontro disso, defende-se que a rede de atenção às pessoas que usam drogas não deve ser considerada apenas em seu aspecto estrutural, envolvendo serviços e profissionais, mas também conforme as interações estabelecidas pelos mesmos. Dessa forma, é necessário que se vá além da identificação dos serviços, procurando entender quais são os processos e as relações que constituem entre si. $^{7-8}$

Ademais, estudos apontam que as redes de atenção aos usuários de drogas são marcadas pela centralização nos serviços especializados, dificuldades de articulação e comunicação, pouca interatividade com dispositivos socioculturais e intervenções assistenciais focadas em situações de urgência e emergência. Tais achados encontram-se na contramão do que é preconizado pela Reforma Psiquiátrica Brasileira, que preza pela territorialidade, descentralização e produção de bem-estar e autonomia. ${ }^{9-10}$

Diante do exposto, compreende-se a complexidade do trabalho neste contexto, e percebese a necessidade do desenvolvimento de metodologias que avaliem a configuração das redes. O uso da Análise de Redes Sociais (ARS) como método pode contribuir significativamente para o desvelamento desse contexto, mostrando que uma rede não se resume à simples soma das 
relações, mas depende do contexto e da posição dos atores dentro dela. A compreensão desses fluxos pode fornecer informações valiosas à tomada de decisões de gestores. ${ }^{11}$

Assim, observa-se a expansão do uso desta metodologia em diversos campos, e a ARS possui características singulares. As medidas utilizadas pela ARS são diversas e devem ser aplicadas de acordo com o propósito do estudo e as características da rede que se pretende analisar. ${ }^{7}$

Frente ao exposto, formulou-se a seguinte questão de pesquisa: "Como é composta a rede social de um Centro de Atenção Psicossocial para álcool e outras drogas (CAPS AD) a partir dos encaminhamentos e recebimento de casos?”. Com base neste questionamento, objetivou-se investigar a rede social de um CAPS AD a partir dos encaminhamentos e recebimentos de casos.

\section{Método}

Trata-se de um estudo quantitativo, exploratório-descritivo, realizado no CAPS AD de um município de médio porte da fronteira oeste do Estado do Rio Grande do Sul, Brasil. O serviço investigado possuía aproximadamente 672 prontuários abertos, dentre estes, em média 30 estavam classificados como tratamento intensivo. Atendia diariamente cerca de 50 a 60 usuários, em atividades individuais ou grupais.

A equipe multiprofissional do serviço era formada por 19 trabalhadores de diversas categorias de nível médio, técnico e superior: agentes sociais, assistente social, enfermeiros, fisioterapeuta, médico psiquiatra, nutricionista, pedagoga, psicólogos, professor de educação física, terapeuta ocupacional e técnicos em enfermagem. Além destes profissionais, contava com residentes em saúde mental e coletiva e acadêmicos de diferentes cursos de graduação da área da saúde.

Os dados foram coletados no período entre agosto e dezembro de 2017. A coleta de dados teve três estágios, aplicados à utilização da ARS na investigação de sistemas de saúde, conforme dados no Quadro $1 .^{12}$ 
Quadro 1 - Estágios de análise de rede social aplicados à investigação de sistemas de saúde

\section{Estágio 1 - Definição da lista de membros e atores da rede:}

Etapa 1: Listagem de todos os stakeholders (grupos de interesse) envolvidos com o CAPS AD e rede de atenção estudados;

Etapa 2: Complementar a lista dos atores com informações coletadas por meio de entrevistas com os informantes-chave.

Estágio 2 - Definição da relação entre os atores:

Etapa 1: Exibir a lista de atores em uma tabela;

Etapa 2: Entrevista com informantes-chave para identificar a relação entre os atores;

Etapa 3: Indicar no quadro a existência ou ausência de relação entre os atores. Em cada quadrado da tabela, coloca-se um "0" quando não houver fornecimento e não houver demanda de informações entre dois atores.

Estágio 3 - análise da estrutura do sistema: mensuração das propriedades (medidas) da rede com ajuda do software Ucinet versão 6.0.

Fonte: adaptação de Blanchet e James, 2011.

No Estágio 1, foi utilizada a técnica de observação sistemática, que teve duração de quatro semanas, objetivando-se a familiarização junto ao CAPS AD, abarcando suas rotinas e fluxos, bem como a aproximação com os informantes-chave da pesquisa. Também foram verificados dados provenientes de documentos e arquivos existentes no serviço, como: fluxogramas, protocolos internos e fichas de atendimento. O uso destas fontes foi útil para explorar a dinâmica das relações estabelecidas pelo CAPS AD.

Ainda no primeiro estágio, realizou-se uma roda de conversa com 13 profissionais, sendo eles: uma assistente social, dois agentes sociais, dois enfermeiros, uma nutricionista, três psicólogos, um professor de educação física e três técnicos em enfermagem. Essa ação visou conhecer quem eram os outros atores com os quais o serviço estabelecia laços relacionais, ou seja, quais as conexões realizadas para resolução de demandas do cotidiano de trabalho relativo aos usuários de drogas. O critério de inclusão nesta etapa foi: profissionais que atuavam no CAPS AD. Foram excluídos do estudo profissionais que estivessem cobrindo licença ou gozando férias no período da coleta de dados. A roda teve duração de aproximadamente 45 minutos, 
Análise da rede social de um Centro de Atenção Psicossocial Álcool e Drogas | 6

tendo sido gravada em áudio e posteriormente transcrita, para facilitar a análise dos dados. Salienta-se que o agente social tem função de orientar e encaminhar aos serviços de assistência social e saúde indivíduos que estejam em vulnerabilidade social.

No estágio 2, aplicou-se um questionário, em formato de Escala Likert, com a finalidade de identificar a relação entre os atores da rede. Foram elaboradas cinco questões, que tinham como conteúdo-chave: referência de envio, referência de recebimento, coordenação de caso, programas conjuntos e consultorias. Cada questão continha a seguinte correspondência de pontuação: 0 - nunca; 1 - raramente; 2 - às vezes; 3 - frequentemente; e 4 - sempre. Este tipo de instrumento consiste de uma série de afirmações a respeito de um determinado objeto. Para cada afirmação há uma escala de zero até 4 pontos.

Nesta etapa, incluíram-se gestores que atuavam na rede de saúde mental do município. Foram excluídos gestores que estivessem assumindo interinamente o cargo de coordenação do serviço ou estivessem cobrindo licença ou gozando férias no período da coleta de dados. A escala foi aplicada separadamente aos gestores (informantes-chave) de cada um dos 37 serviços (chamados aqui de atores da rede) indicados pelos trabalhadores do CAPS AD durante o primeiro estágio da coleta de dados. O valor da medida é obtido por meio da soma dos valores das respostas às afirmações.

Este estudo enfoca dois aspectos que identificam os modos de relacionamento estabelecidos entre os atores, a saber: ${ }^{13}$

a) Referência de envio: pacientes enviados a outras unidades da rede;

b) Referência de recebimento: pacientes recebidos de outras unidades da rede.

De posse desses dados, partiu-se para o estágio três, no qual foram construídos sociogramas e realizou-se a mensuração das propriedades (medidas) da rede - Densidade e Centralidade, constituindo-se, portanto, o $3^{\text {}}$ Estágio. $^{12}$ 
Destaca-se aqui o uso das métricas de Densidade e Centralidade. O objetivo da Densidade é medir o grau de conectividade dentro da rede, representando o número de ligações possíveis totais que podem existir. Uma rede é considerada densa quando apresenta muitas relações entre pares de atores; caso contrário, é pouco densa. ${ }^{10-11}$ Já o Grau de Centralidade permite obter o grau de influência do ator em relação à rede local.

Para a composição dos sociogramas, foi utilizado um software de computador chamado Ucinet. Trata-se de uma representação gráfica da rede, na qual cada ator é um ponto e as linhas que os ligam representam as relações entre eles. O Ucinet calcula a distância geodésica entre os atores, isto é, o número e a intensidade das conexões entre atores para estabelecer a posição e a proximidade.

Para a realização deste estudo foram respeitados os aspectos éticos e legais pautados na Resolução n 466, de 12 de dezembro de 2012, do Conselho Nacional de Saúde, que regulamenta a pesquisa envolvendo seres humanos. A aprovação do Comitê de Ética em Pesquisa da Universidade Federal do Rio Grande do Sul ocorreu em 27 de julho de 2017 e foi registrada sob o número 2.189.159.

\section{Resultados}

Os resultados apontaram a existência de 37 atores envolvidos no cuidado às pessoas que usam drogas. É possível observar que se trata de uma rede com dispositivos da saúde, assistência social, outros setores e recursos comunitários/equipamentos sociais, de natureza generalista e especializada (saúde mental e álcool e outras drogas), e de distintos níveis de atenção/complexidade e finalidades. A representação também inclui serviços de caráter público e privado, instituições filantrópicas e organizações não governamentais (ONGs), conforme demonstra o Quadro 2. 
Análise da rede social de um Centro de Atenção Psicossocial Álcool e Drogas | 8

Quadro 2 - Serviços que fazem parte da Rede de Cuidado às pessoas que usam drogas. Uruguaiana/RS, Brasil, 2018

\begin{tabular}{|c|c|}
\hline \multicolumn{2}{|r|}{ Componentes da RAPS } \\
\hline \multirow{2}{*}{ Atenção Básica em Saúde } & Estratégia de Saúde da Família (ESF) \\
\hline & Consultório na Rua $(\mathrm{CnR})$ \\
\hline \multirow{2}{*}{$\begin{array}{l}\text { Atenção Psicossocial } \\
\text { Estratégica }\end{array}$} & CAPS AD \\
\hline & CAPS II \\
\hline \multirow{3}{*}{$\begin{array}{l}\text { Atenção de Urgência e } \\
\text { Emergência }\end{array}$} & Unidade de Pronto Atendimento (UPA) \\
\hline & Pronto Atendimento (Hospital) \\
\hline & Ambulatório de Saúde Mental \\
\hline Atenção Hospitalar & Unidade de Psiquiatria (Hospital) \\
\hline \multirow{2}{*}{$\begin{array}{l}\text { Atenção Residencial de } \\
\text { Caráter Transitório }\end{array}$} & Casa de Passagem \\
\hline & Comunidade Terapêutica \\
\hline $\begin{array}{l}\text { Estratégias de Reabilitação } \\
\text { Psicossocial }\end{array}$ & Sistema Nacional de Empregos (SINE) \\
\hline \multicolumn{2}{|r|}{ Atores além da RAPS - Recursos intersetoriais } \\
\hline \multirow{21}{*}{$\begin{array}{l}\text { Saúde/ } \\
\text { Educação/ } \\
\text { Justiça/ } \\
\text { Assistência Social }\end{array}$} & Centro de Orientação e Apoio Sorológico (COAS) \\
\hline & Farmácia Popular \\
\hline & Centro de Administração de Medicações Injetáveis (CAMI) \\
\hline & Projeto de Saúde Prevenção e Agravos de Doenças não Transmissíveis (DANTS) \\
\hline & Escolas \\
\hline & Universidade Federal do Pampa (UNIPAMPA) \\
\hline & Secretaria de Esporte \\
\hline & Casa Flores de Maria - Moradia de Idosos (Lar/moradia de caráter privado) \\
\hline & Casa de Acolhimento a Criança e Adolescente de Uruguaiana (CACAU) \\
\hline & Centro de Referência Especializado da Assistência Social (CREAS) \\
\hline & Centro de Referência da Assistência Social (CRAS) \\
\hline & Promotoria/Defensoria Pública \\
\hline & Delegacia da Mulher \\
\hline & Polícia Civil \\
\hline & Ronda Ostensivas Municipais (ROMU) \\
\hline & Brigada Militar \\
\hline & Fundação de Atendimento Socioeducativo (FASE) \\
\hline & Penitenciária Modulada \\
\hline & Conselho Tutelar \\
\hline & Conselho Municipal de Saúde \\
\hline & Conselho Municipal Sobre Drogas (COMAD) \\
\hline \multirow{5}{*}{ Equipamentos Sociais } & Entidade de apoio às pessoas com HIV (GUAPA) \\
\hline & Amor Exigente \\
\hline & Alcoólicos Anônimos (AA) \\
\hline & Narcóticos Anônimos (NA) \\
\hline & Associação de Familiares \\
\hline
\end{tabular}

Rev. Enferm. UFSM, Santa Maria, v10, p. 1-20, 2021 
9 | Siniak DS, PinhoII LB, Júnior JNM, Ávila MB, Silva VAM

O uso da ARS identifica as relações entre os atores e, a partir daí, estabelece proximidades ou distanciamentos por meio das distâncias geodésicas. O sociograma apresentado a seguir (Figura 1) demonstra graficamente esta visão, na perspectiva da Referência de Envio.

Figura 1 - Sociograma da rede na perspectiva de referência de envio, Uruguaiana/RS, Brasil, 2017

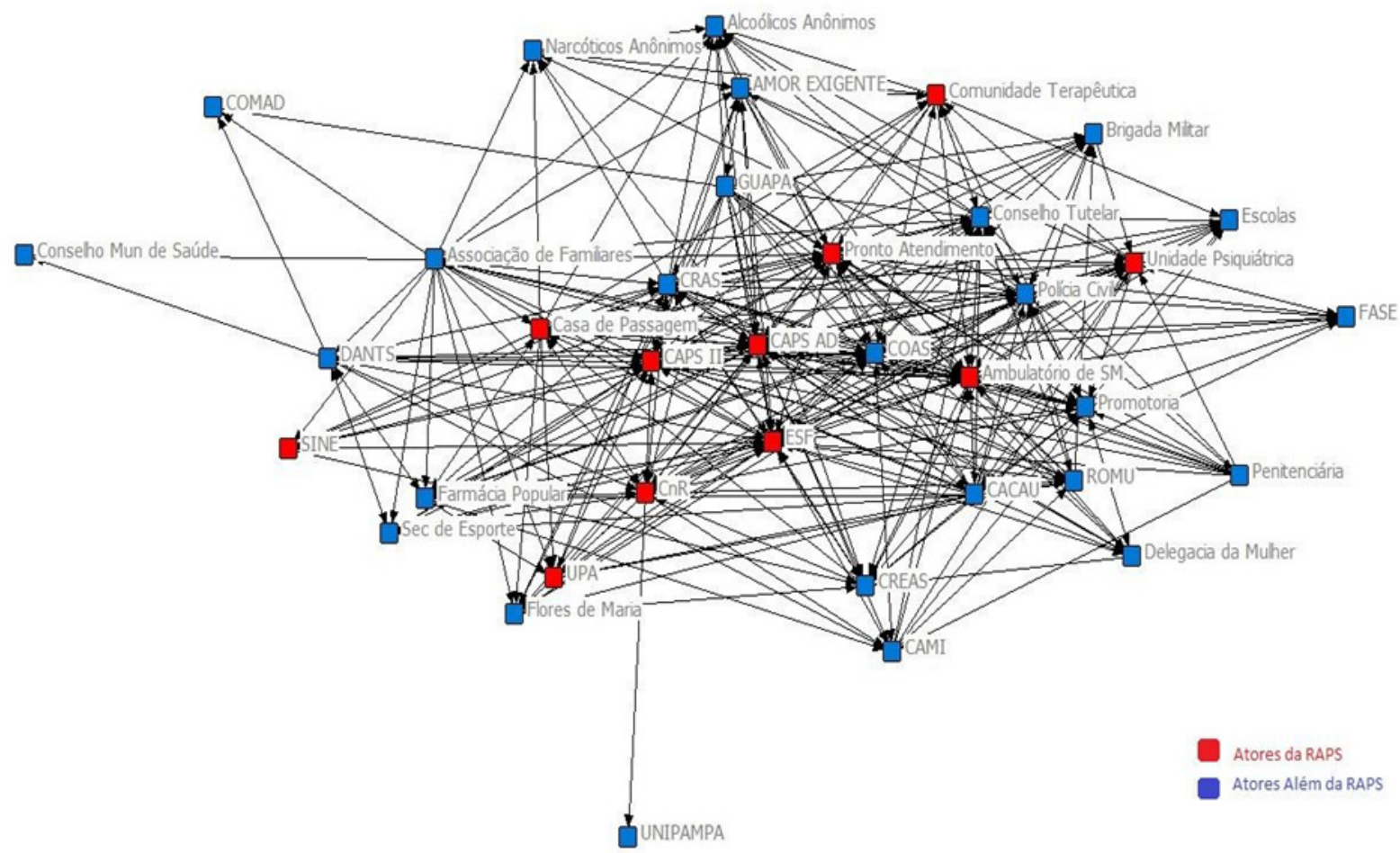

$\mathrm{Na}$ rede investigada, cada um dos serviços representados pode enviar pacientes para as outras 36 unidades da rede. Essa configuração resulta em um potencial de 1.332 conexões. Os resultados indicam que, no tocante à referência de envio de pacientes, a rede apresentou 374 conexões. Portanto, a densidade média dessa rede, dividindo-se esse valor pelo potencial total de conexões, foi de $28,1 \%$, o que caracteriza uma rede de baixa densidade. Em contrapartida, quando verificada a densidade média apenas entre os atores que fazem parte da RAPS (11 unidades), identificou-se um percentual de 53,6\%. 
Com relação ao grau de Centralidade da rede, identificou-se que o CAPS AD assumia o papel central nas relações de envio, fazendo 71 conexões com os demais atores. Outros dispositivos que podem ser considerados centrais no envio de pacientes foram: CRAS (56), Conselho Tutelar (48), GUAPA (46) e CACAU (45). Os atores UNIPAMPA, Conselho Municipal de Saúde e COMAD não efetuaram conexões com outros atores da rede no tocante à relação de envio de pacientes, o que, no sociograma (Figura 1), evidencia-se pela posição ocupada por estes serviços, distantes do centro.

A perspectiva de análise de Referência de Recebimento, por sua vez, apresentou um número ligeiramente menor que a de Envio, sendo possível observar na Figura 2 uma dinâmica menos intensa de trocas entre os atores.

A densidade média total da rede de recebimento indica que a referência de recebimento de pacientes apresentou 300 conexões; valor que, dividido pelo potencial total de conexões na rede anteriormente mencionado (1.332 conexões), resulta em uma rede com densidade média de $22,5 \%$, o que caracteriza uma rede com densidade menor que a de referência de envio. Com relação à densidade média, apenas entre os 11 atores que compõem a RAPS, verificou-se novamente uma densidade maior que a da rede geral, com 58,8\%. A Figura 2 ilustra a configuração dessa rede.

Figura 2 - Sociograma da rede na perspectiva da Referência de Recebimento Uruguaiana/RS, Brasil, 2017 


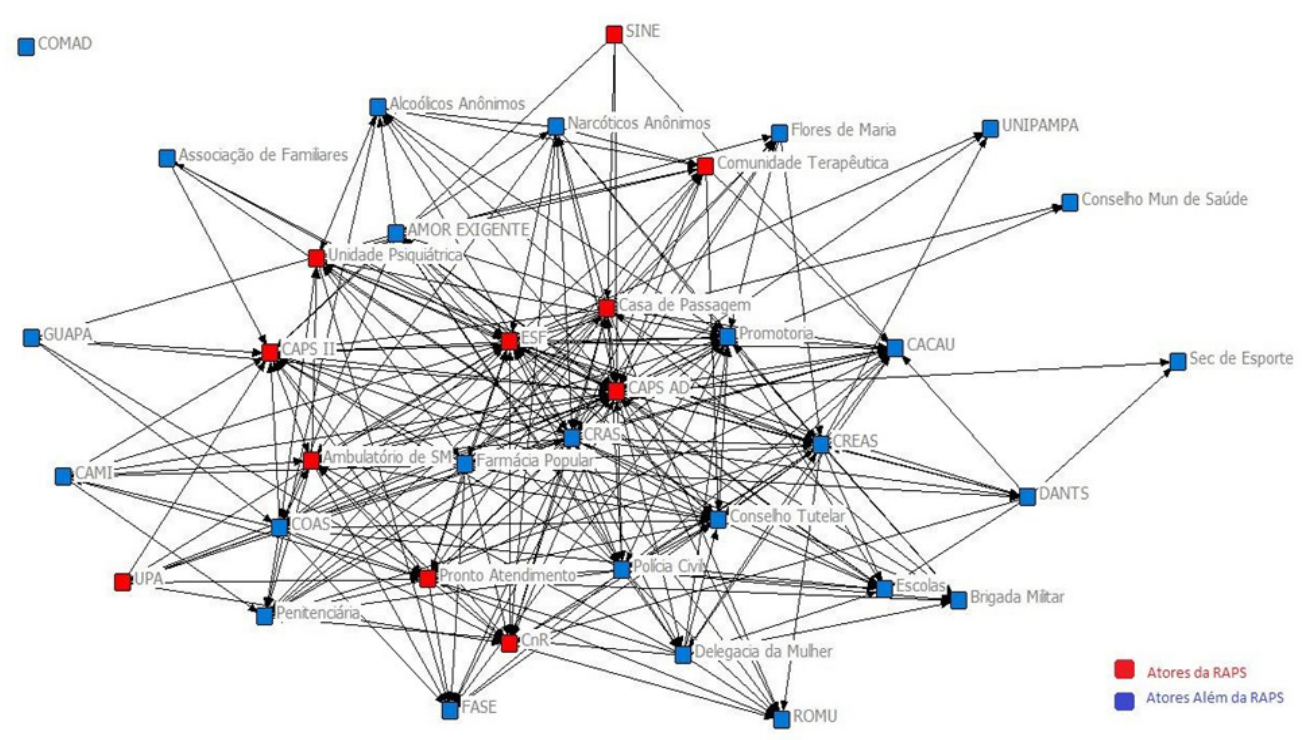

Com relação ao grau de Centralidade da Rede de recebimento, identificou-se que os três atores que possuíam maior número de conexões com relação ao recebimento de pacientes eram: CAPS AD (115), ESF (50) e CRAS (47). Já os atores Escolas, Secretaria de Esporte, ROMU, FASE, Penitenciária e COMAD não estabeleceram conexões nesse modo de relacionamento.

\section{Discussão}

A complexidade do fenômeno das drogas revela inúmeras necessidades relacionadas às perspectivas sociais precárias, falta oportunidade de moradia, saúde, emprego e desenvolvimento pessoal/profissional; com histórias de vida permeadas por diferentes tipos de violência, históricos familiares com uso abusivo e dependentes de drogas, dentre outros fatores de risco socioculturais e ambientais. ${ }^{13-14}$ Diante disso, o cuidado aos usuários dos serviços também exige intervenções por meio de estratégias multifatoriais. ${ }^{15-16}$

Os resultados mostram que os serviços que compõem a RAPS estabelecem um maior número de relações entre si em comparação com o restante da rede, o que pode ser considerado um padrão esperado de laços entre os atores. Esta conectividade pode ser evidenciada na relação de envio (encaminhamento) e de recebimento de usuários dos serviços, onde o grau de densidade entre os 11 atores da RAPS foi maior que o da rede como um todo. No entanto, quando a 
densidade total da rede é analisada, ou seja, quando consideradas somente as ligações existentes entre os 37 atores, verificou-se um índice de $28,1 \%$ e $22,5 \%$ nas relações de envio e recebimento de usuários, respectivamente.

Sabe-se que a rede criada para usuários de drogas teve sua implementação interpelada por orientações antidrogas, onde o uso de drogas foi encarado como um problema de segurança pública e não como um assunto sanitário e de cuidados com os mesmos. Desta forma, ressaltase, aqui, o desafio e a importância da articulação entre os atores da RAPS para garantia da qualidade do cuidado prestado, pois as redes que cuidam podem cercar esta população. ${ }^{17}$

Um cuidado efetivo depende de ações que valorizem a articulação com diferentes recursos para o desenvolvimento de suas práticas. Assim, compreende-se que o processo de cuidado às pessoas que usam drogas deve estar ancorado na perspectiva de formação de parcerias com diferentes setores e serviços disponíveis no território, para além dos setores que compõem tradicionalmente a RAPS, e dialogando com os recursos comunitários do território de origem das pessoas acessadas.

Nesse sentido, a rede de cuidado deve ser composta por pontos plurais que busquem desmitificar a hegemonia do setor da saúde, por intermédio da valorização das complexidades dos contextos socioculturais e das comunidades. E por meio do aumento da articulação e participação de espaços de educação, cultura, cidadania, trabalho, lazer, espiritualidade e transformação social. ${ }^{18}$ Desse modo, recursos comunitários tornam-se fundamentais para a promoção, prevenção e reinserção social de usuários de drogas, sendo lugares e espaços onde se encontra a vida como ela é, em que as relações e os laços são desvelados. ${ }^{13}$

A existência desses diferentes níveis de relacionamento dentro da rede é benéfica do ponto de vista da descentralização e ampliação da assistência. Encaminhamentos entre serviços são fatores que mostram certa comunicabilidade entre eles, bem como um funcionamento que 
vise à garantia do cuidado centrado nas demandas da pessoa acessada de forma integral, em todas as suas dimensões.

No entanto, o foco nos sistemas de encaminhamentos, referência e contrarreferência pode contribuir para a tenuidade da articulação e fluidez da rede e da integralidade assistencial. Pois é necessário o investimento em estratégias de atenção pautadas em corresponsabilização, cuidado compartilhado e apoio matricial. Visando, assim, a superação de modelos assistenciais fragmentados e a transversalização do cuidado em saúde mental. ${ }^{19}$

O CAPS AD é um dos principais serviços que encaminha demandas relacionadas às pessoas que usam drogas, reforçando-se a constatação de que há uma demanda integral por parte desse público para a garantia e efetividade dos projetos terapêuticos. O fato de constituirse como um serviço de referência não implica na efetivação do cuidado de forma isolada; pelo contrário, é papel desta referência identificar as diferentes demandas, buscando articulação junto aos demais dispositivos da rede.

Sabe-se que muitos usuários do serviço apresentam diferentes problemas clínicos de saúde e vulnerabilidades sociais, necessitando de cuidados específicos e constantes de outros dispositivos assistenciais. Contudo, o CAPS AD era o único serviço acessado por eles. Assim, compreende-se que esta forma de organização da rede pode gerar sobrecarga ao serviço, além de impactar negativamente no cuidado, numa concentração de demandas que tende a limitar a potencialidade da rede.

Dessa forma, o CAPS AD não deve constituir-se em um serviço centralizador e totalitário. Visto que garantir o acesso dos usuários a diferentes pontos da rede é parte integrante do cuidado holístico e singular e dos ideais da Reforma Psiquiátrica que almejam a inserção social, as ações extramuros, a articulação com o território, o respeito às singularidades, a liberdade e a autonomia. ${ }^{20}$ 
O Conselho Tutelar e CACAU também figuraram entre os serviços que mais realizavam encaminhamentos. Essa interface acontece em muitos casos devido a questões intrafamiliares que possam, por exemplo, impedir temporariamente que pais permaneçam com a guarda dos filhos. Em outros casos, esses dispositivos também são acionados por conta da exposição de crianças e adolescentes a situações de risco relacionadas ao fluxo clandestino de drogas em suas comunidades.

Esse achado reforça outros resultados encontrados sobre as implicações da atenção psicossocial e da institucionalização no atendimento às necessidades de adolescentes em situação de uso de drogas ilícitas, bem como às de seus familiares. ${ }^{14}$ Nesses estudos evidenciouse a necessidade de fortalecimento da RAPS e sua articulação intersetorial, considerando que as ações de promoção e prevenção relacionadas ao uso de drogas voltadas aos adolescentes e seus familiares ainda são frágeis. E as estratégias de cuidado muitas vezes são enfatizadas na repressão, no combate e na hipermedicalização. ${ }^{14,21}$

No caso do Conselho Tutelar, é importante que este ator possa oferecer um espaço de acolhimento e escuta, e que seja capaz de compreender as diferentes demandas sociais dentro da comunidade, visto que pode ser considerado como uma das primeiras "portas de entrada" para atendimento de demandas relacionadas ao uso de drogas na rede. ${ }^{13}$ Neste caso, o Conselho Tutelar para o usuário torna-se um espaço de garantia de direitos humanos e sociais, que permite melhorar sua qualidade de vida e bem-estar e buscar alternativas para solucionar seus problemas, tanto nos aspectos sociais quanto econômicos, culturais, entre outros. ${ }^{22}$

Chama a atenção a expressividade do GUAPA, que apareceu como um serviço relevante na relação de envio dentro da rede. Isso demonstra alguma articulação entre recursos comunitários e a rede de atenção formal, como um elemento enriquecedor para a construção de possibilidades de cuidado, conectadas ao território existencial dos sujeitos acessados.

Portanto, compreende-se que uma rede ampliada, formada não apenas por serviços de saúde, mas indivíduos, equipamentos do território e organizações, ofertará uma assistência 
ampliada, de modo a promover a inclusão de múltiplos setores e a integralidade das ações..$^{5,7} \mathrm{O}$ CRAS e o CREAS também aparecem como dispositivos centrais nas referências, reforçando a relevância do Sistema Único de Assistência Social no atendimento efetivo a estas demandas.

Considera-se necessário olhar para a compreensão da temática não somente como um problema em si, ou puramente no plano individual e biológico, mas levando em conta o papel das estruturas sociais na conformação dos sujeitos e seus determinantes sociais, ou seja, como elementos que compõem os fatores de risco e de proteção envolvidos nos problemas associados ao uso de álcool e outras drogas. ${ }^{8,23-24}$

A atenção básica $(\mathrm{AB})$ e as equipes da Estratégia de Saúde da Família (ESF) também despontam no encaminhamento e recebimento de casos. Enquanto a ESF figura entre os principais dispositivos que favorecem os encaminhamentos de casos, ela também aparece como o segundo serviço da rede que mais recebe demandas vindas de outros serviços, por estar inserida no território.

A $A B$ é responsável por oferecer à população os cuidados necessários para os seus problemas de saúde mais prevalentes, incluindo medidas preventivas, curativas, de reabilitação e promoção de saúde, devendo ser resolutiva para cerca de $80 \%$ destes problemas; sobretudo, é responsável pela continuidade e integralidade da atenção e da coordenação da assistência dentro do próprio sistema, além do fato de ser vista como um ambiente com menor probabilidade de promover preconceito e estigma..$^{25}$

Contudo, estudos apontam a baixa capacidade de resolutividade das unidades da ESF, não devido ao potencial que apresentam, mas relacionada a problemas estruturais e de recursos humanos, altas demandas e sobrecarga, contribuindo para fortalecer a cultura do encaminhamento. ${ }^{23-24}$ Assim, quando estes dispositivos encontram dificuldades, encaminham os casos aos serviços especializados, estabelecendo uma relação de dependência com os mesmos, ao mesmo tempo diminuindo-se não só o alcance de intervenções preventivas, educativas e de 
promoção da saúde, bem como o acompanhamento de casos que já tenham recebido alta de internações hospitalares ou dos CAPS, dificultando a corresponsabilização do cuidado com os parceiros de áreas especializadas. ${ }^{25}$

A qualificação insuficiente dos profissionais na $A B$ pode resultar em dificuldades de planejamento do cuidado, assim como no reforço à lógica reducionista e estigmatizadora no cuidado às pessoas acessadas. Por outro lado, a capacitação desses profissionais, aliada à responsabilidade compartilhada entre os serviços, contribui para a construção de movimentos de ruptura com o modelo tradicional, individual e especializado de atenção à saúde. ${ }^{26}$

Reconhece-se que as demandas dos usuários dos serviços são diversas e, por isso, exigem a cooperação de diferentes serviços para efetivação do cuidado. Isso quer dizer que o encaminhamento se faz necessário e vital em muitos casos, contudo, esta prática não deve se resumir em uma transferência do cuidado. Ao contrário, deve-se investir na responsabilização partilhada e na comunicação entre os serviços envolvidos. ${ }^{15-17,25}$ Desse modo, considera-se que a organização da rede de atenção deve estar integrada a uma gama de recursos e funcionar de forma articulada, valorizando a importância de cada serviço dentro da rede.

Como limitação de estudo, tem-se que o alcance desta pesquisa possibilita apenas respostas parciais ao fenômeno avaliado, pois uma realidade foi detalhada e estudada.

\section{Conclusão}

A investigação da rede social de um CAPS AD, a partir dos encaminhamentos e recebimentos de casos, apontou a existência de relações entre importantes atores da rede. Destaca-se a importância dos serviços ligados à RAPS, assim como a parceria com outros serviços e setores nessa composição.

Analisar a rede social do CAPS AD possibilita ampliar a assistência de cuidado e diminuir a sobrecarga dos serviços, sendo a articulação um ponto fundamental para visualizar as 
potencialidades de cada serviço. Ressalta-se que, embora o CAPS AD seja um dos principais encaminhadores das demandas relacionadas às pessoas que usam drogas, é fundamental o relacionamento dos locais que atendem essa demanda.

Sugere-se o desenvolvimento de novos estudos com a utilização de outros métodos de análise, inclusive qualitativos, e realizados em outros contextos, para explicação de fenômenos inerentes ao cotidiano dos serviços e de funcionamento das equipes.

\section{Referências}

1. Ministério da Justiça e Cidadania (BR), Secretaria Nacional de Políticas sobre Drogas. Módulo 1: o uso de substâncias psicoativas no Brasil [Internet]. Brasília (DF): Ministério da Justiça e Cidadania; 2017 [acesso em 2020 out 30]. Disponível em: https://www.supera.org.br/@/material/mtd/pdf/SUP/SUP_Mod1.pdf

2. United Nations Office on Drugs and Crime (UNODC). World Drug Report 2018 [Internet]. New York: UNODC; 2018 [cited 2020 Oct 30]. Available from: https://www.unodc.org/wdr2018/

3. Mateus MD, organizador. Políticas de saúde mental: baseado no curso Políticas públicas de saúde mental, do CAPS Professor Luiz da Rocha Cerqueira [Internet]. São Paulo: Secretaria de Estado da Saúde de São Paulo; 2013 [acesso em 2020 out 30]. Disponível em: https://www.researchgate.net/profile/Denise_Razzouk/publication/286916533_Economia_em_Saude_Men tal_Mental_Health_Economics/links/566f303e08ae486986b701a2/Economia-em-Saude-Mental-MentalHealth-Economics.pdf

4. Camatta MW, Siniak DS, Schneider JF, Pinho LB, Eslabão AD, Ribeiro DB. Concepções de gestores de serviços de saúde sobre a rede de atenção psicossocial a usuários de crack. REME Rev Min Enferm [Internet]. 2020 [cited 2020 Oct 30];24:e-1293. Available from: https://www.reme.org.br/artigo/detalhes/1439

5. Eslabão AD, Coimbra VCC, Kantorski LP, Pinho LB, Santos EO. Mental health care network: the views of coordinators of the Family Health Strategy (FHS). Rev Gaúcha Enferm. 2017;38(1):e60973. doi: https://doi.org/10.1590/1983-1447.2017.01.60973

6. Costa PHA, Martins LF, Medeiros AX, Salgado JA, Silva WMD, Ronzani TM, et al. Referral and counter-referral system in the care network for drug users: contributions from social network analysis. Cad Saúde Coletiva. 2015;23(3):245-52. doi: https://doi.org/10.1590/1414-462X201500030129

7. Costa PHA, Mota DCB, Paiva FS, Ronzani TM. Desatando a trama das redes assistenciais sobre 
Análise da rede social de um Centro de Atenção Psicossocial Álcool e Drogas | 18

drogas: uma revisão narrativa da literatura. Ciênc Saúde Colet. 2015;20(2):395-406. doi: https://doi.org/10.1590/1413-81232015202.20682013

8. Milward HB, Provan KG. Measuring network structure. Public Administr. 2002;76(2):387-407. doi: https://doi.org/10.1111/1467-9299.00106

9. Costa PHA, Ronzani TM, Colugnati FAB. No meio do caminho tinha um CAPSAD: centralidade e lógica assistencial da rede de atenção aos usuários de drogas. Ciênc Saúde Colet. 2018;23(10):3233-45. doi: https://doi.org/10.1590/1413-812320182310.12572018

10. Dantas NF, Passos ICF. Apoio matricial em saúde mental no SUS de Belo Horizonte: perspectiva dos trabalhadores. Trab Educ Saúde. 2018;16(1):201-20. doi: 10.1590/1981-7746-sol00097

11. Silveira MAP, Farina MC. Análise de redes sociais como ferramenta que contribui para a melhoria das relações entre empresas participantes de um APL de eventos. Redes (St Cruz Sul) [Internet]; 2012 [acesso em 2020 out 30];17(1):33-54. Disponível em: https://online.unisc.br/seer/index.php/redes/article/view/2384

12. Sabot K, Wickremasinghe D, Blanchet K, Avan B, Schellenberg J. Use of social network analysis methods to study professional advice and performance among healthcare providers: a systematic review. Syst Rev. 2017;6(208). doi: https://doi.org/10.1186/s13643-017-0597-1

13. Pinho LB, Wetzel C, Schneider JF, Olschowsky A, Camatta MW, Kohlrausch ER, et al. Evaluation of intersectoral resources in the composition of care networks for crack users. Esc Anna Nery. 2017;21(4):e20170149. doi: https://doi.org/10.1590/2177-9465-ean-2017-0149

14. Paula ML, Jorge MSB, Lima LL, Bezerra IC. Experiences of adolescent crack users and their relatives with psychosocial care and institucionalization. Ciênc Saúde Coletiva. 2017;22(8):2735-44. doi: https://doi.org/10.1590/1413-81232017228.22892015

15. Siniak DS, Pinho LB. Emotional support received by families state of the crack users. Rev Enferm UFPE Online [Internet]. 2015 [cited 2020 Oct 30];9(3):7656-63. Available from: https://periodicos.ufpe.br/revistas/revistaenfermagem/article/view/10505

16. Claus MIS, Zerbetto SR, Gonçalves AMS, Galon T, Andrade LGZ, Oliveira FC. As forças familiares no contexto da dependência de substâncias psicoativas. Esc Anna Nery. 2018;22(4):e20180180. doi: https://doi.org/10.1590/2177-9465-EAN-2018-0180

17. Pinho LB, Wetzel C, Schneider JF, Olschowsky A, Camatta MW, Kohlrausch ER, et al. Assessment of components of crack users' attention network. Rev Bras Enferm. 2020;73(1):e20170835. doi: https://doi.org/10.1590/0034-7167-2017-0835

18. Silva LM, Olschowsky A, Silva AB, Pavani FM, Wetzel C. Ações de intersetorialidade em saúde mental: uma revisão integrativa. Rev Pesq Cuid Fundam. 2019;11(3):763-70. doi: 
19 | Siniak DS, PinhoII LB, Júnior JNM, Ávila MB, Silva VAM

https://doi.org/10.9789/2175-5361.2019.v11i3.763-770

19. Lima MC, Gonçalves TR. Apoio matricial como estratégia de ordenação do cuidado em saúde mental. Trab Educ Saúde. 2020;18(1):e0023266. doi: https://doi.org/10.1590/1981-7746-sol00232

20. Campos DB, Bezerra IC, Jorge MSB. Produção do cuidado em saúde mental: práticas territoriais na rede psicossocial. Trab Educ Saúde. 2020;18(1):e0023167. doi: https://doi.org/10.1590/1981-7746-sol00231

21. Galhardi CC, Matsukura TS. O cotidiano de adolescentes em um Centro de Atenção Psicossocial de Álcool e outras Drogas: realidades e desafios. Cad Saúde Pública. 2018;34(3):e00150816. doi: https://doi.org/10.1590/0102-311x00150816

22. Romagnoli RC, Silva BC. A inserção do Conselho Tutelar na prática da intersetorialidade. Gerais (Univ Fed Juiz Fora). 2019;12(2). doi: http://dx.doi.org/10.36298/gerais2019120203

23. Costa PHA, Paiva FS. Revisão da literatura sobre as concepções dos profissionais de saúde sobre o uso de drogas no Brasil: modelo biomédico, naturalizações e moralismos. Physis. 2016;26(3):1009-31. doi: https://doi.org/10.1590/s0103-73312016000300015

24. Pettersen H, Landheim A, Skeie I, Biong S, Brodahl M, Oute J, et al. How social relationships influence substance use disorder recovery: a collaborative narrative study. Subst Abuse. 2019;9(13):1-8. doi: https://doi.org/10.1177/1178221819833379

25. Wenceslau LD, Ortega F. Mental health within primary health care and Global Mental Health: international perspectives and Brazilian context. Interface (Botucatu). 2015;19(55):1121-32. doi: https://doi.org/10.1590/1807-57622014.1152

26. Varela DSS, Sales IMM, Silva FMD, Monteiro CFS. Health network assisting users of alcohol, crack, and other drugs. Esc Anna Nery. 2015;20(2):296-302. doi: https://doi.org/10.5935/1414-8145.20160040

Editor Científico: Tânia Solange Bosi de Souza Magnago

Editor Associado: Daiana Foggiato de Siqueira

\section{Autor correspondente}

Marciele Barcelos Ávila

E-mail: marcieleba@yahoo.com.br

Endereço: R. São Manoel, 963 - Rio Branco, Porto Alegre.

CEP: $90620-110$ 
Análise da rede social de um Centro de Atenção Psicossocial Álcool e Drogas | 20

\section{Contribuições de Autoria:}

\section{1 - Débora Schlotefeldt Siniak}

Concepção ou desenho do estudo/pesquisa/ análise e interpretação dos dados.

\section{2 - Leandro Barbosa de Pinho}

Concepção ou desenho do estudo/pesquisa

\section{3 - João Nunes Maidana Júnior}

Revisão final com participação crítica e intelectual no manuscrito

\section{4 - Marciele Barcelos Avila}

Revisão final com participação crítica e intelectual no manuscrito

\section{5 - Vanessa Alvez Mora da Silva}

Revisão final com participação crítica e intelectual no manuscrito

\section{Como citar este artigo}

Siniak DS, PinhoII LB, Júnior JNM, Avila MB, Silva VAM. Análise da rede social de um Centro de Atenção Psicossocial Álcool e Drogas. Rev. Enferm. UFSM. 2021 [Acesso em: Ano Mês Dia]; vol.10 e32: 1-20. DOI:https://doi.org/10.5902/2179769248177 